\title{
EQUIVALENCE-SINGULARITY DICHOTOMIES FROM ZERO-ONE LAWS ${ }^{1}$
}

\author{
RAOUL D. LEPAGE AND V. MANDREKAR
}

ABSTRACT. In this note a general result on equivalence and singularity of two measures is presented. As a consequence of this S. Kakutani's dichotomy for product measures and J. Feldman's dichotomy for Gaussian measures are derived via appropriate zero-one laws.

In several different contexts, probability measures $P, Q$ which are mutually absolutely continuous on each $\mathscr{F}_{n}$ of an increasing sequence of $\sigma$-algebras $\left\{\mathscr{F}_{n}, n=1,2, \cdots\right\}$ are then necessarily mutually absolutely continuous $(\equiv)$ or singular $(\perp)$ on the minimal $\sigma$-algebra containing $\bigcup_{n} \mathscr{F}_{n}$. Invariably, some type of 0-1 law is operative and suspected of forcing the equivalence-singularity dichotomy.

Here it is shown how in each case the dichotomy results from a certain tail $\sigma$-algebra being trivial. The natural technique for establishing triviality of this tail $\sigma$-algebra is none other than use of the appropriate 0-1 law.

THEOREM. Let $\mathscr{F}_{1} \subset \mathscr{F}_{2} \subset \cdots$ be $\sigma$-algebras of subsets of $\Omega, \mathscr{F}=$ $\sigma\left(\bigcup_{n} \mathscr{F}_{n}\right)$ the $\sigma$-algebra generated by their union. For any probability measures $P, Q$ on $\widetilde{F}$ which are mutually absolutely continuous when restricted to each of $\mathscr{F}_{1}, \mathscr{F}_{2}, \cdots$, we let $\rho_{n}=\left(d Q \mid \mathscr{F}_{n}\right) /\left(d P \mid \mathscr{F}_{n}\right)$ and define the tail algebra $\mathcal{S}=\bigcap_{n} \sigma\left\{\log \rho_{k+1}-\log \rho_{k} \mid k \geqq n\right\}$. The following is then true:

$$
(A \in \mathfrak{S} \Rightarrow P(A)=0 \text { or } 1) \Rightarrow(P \perp Q \text { or } P \ll Q) .
$$

Proof. The measure $Q$ has a Hahn decomposition by $P$ which is

$$
Q(A)=\int_{A} \rho d P+Q(A N) \text { for every } A \in \mathcal{F}
$$

where $\rho$ is a nonnegative $\mathfrak{F}$-measurable function and $N$ is a $P$-negligible $\mathscr{F}$-measurable set. Using the submartingale convergence theorem it has

Received by the editors March 9, 1971.

AMS 1970 subject classifications. Primary 60G30; Secondary 60F20.

Key words and phrases. Singularity, equivalence, product measure, Gaussian measure, zero-one law.

${ }^{1}$ Research was partially supported by NSF Grant GP-11626. 
been proved that $P\left(\lim _{n} \rho_{n}=\rho\right)=1$ (e.g. [1, Lemma 3]). Observe that

$$
Q\left(\rho_{n}=0\right)=\int_{\left(\rho_{n}=0\right)} \rho_{n} d P=0 \text { for every } n
$$

so that by mutual absolute continuity $P\left(\rho_{n}=0\right)=0$ for every $n$. Therefore $(\rho>0)$ belongs to the $P$-completion of $\mathcal{G}$ since a.e. $P$,

$$
\begin{aligned}
(\rho>0) & =\left(\lim _{N} \rho_{N}>0\right)=\left(\lim _{N} \log \rho_{N+1}>-\infty\right) \\
& =\left(\lim _{N} \sum_{k=n}^{k=N}\left(\log \rho_{k+1}-\log \rho_{k}\right)>-\infty\right) \\
& \in \sigma\left\{\log \rho_{k+1}-\log \rho_{k} \mid k \geqq n\right\}
\end{aligned}
$$

for every $n$.

If every event in $\mathcal{G}$ is $P$-trivial, then $P(\rho>0)=0$ or $P(\rho>0)=1$. In the former case

$$
Q(N)=Q(\Omega)-\int_{\Omega} \rho d P=1, \quad P(N)=0
$$

so that $P \perp Q$. In the case $P(\rho>0)=1$, for every $A \in \mathscr{F}$ and $\alpha>0$,

$$
Q(A) \geqq \int_{A} \rho d P \geqq \int_{A(\rho>\alpha)} \rho d P \geqq \alpha P(A(\rho>\alpha))
$$

and $P(A(\rho>\alpha)) \rightarrow P(A)$ as $\alpha \downarrow 0$. Hence $P(A)>0$ implies $Q(A)>0$. That is, $Q(A)=0$ implies $P(A)=0$. Therefore $P \ll Q$.

COROLlary. With the same assumptions as in the Theorem,

$$
(A \in \mathcal{S} \Rightarrow P(A)=0 \text { or } 1 \text { and } Q(A)=0 \text { or } 1) \Rightarrow(P \perp Q \text { or } P \equiv Q) \text {. }
$$

Proof. Interchanging the roles of $P$ and $Q$ does not alter $\mathcal{G}$ or the assumptions of the Theorem.

Each of the two major types of equivalence-singularity dichotomies for measures will now be proved to follow via the Corollary. In every case, our method is the same: Use an appropriately chosen 0-1 law to establish triviality of $\mathcal{G}$ with respect to each of $P$ and $Q$.

KAKUtANi's Theorem. Suppose $(\Omega, \mathfrak{F})=\left(\mathrm{X}_{k=1}^{\infty} \Omega_{k}, \bigotimes_{k=1}^{\infty} \mathfrak{F}^{(k)}\right)$ and $P=\mathrm{X}_{k=1}^{\infty} P^{(k)}, Q=\mathrm{X}_{k=1}^{\infty} Q^{(k)}$. Then

$$
\left(P^{(k)} \equiv Q^{(k)} \text { for every } k\right) \Rightarrow(P \perp Q \text { or } P \equiv Q) .
$$

Proof. For every $n$ let $p^{(n)}=d Q^{(n)} / d P^{(n)}$,

$$
\mathcal{F}_{n}=\bigotimes_{k=1}^{n} \mathcal{F}^{(k)} \otimes \bigotimes_{k=n+1}^{\infty}\left\{\phi, \Omega_{k}\right\},
$$

then $\log \rho_{n+1}-\log \rho_{n}=\log \rho^{(n+1)}$. Hence $\mathcal{S}$ is the tail algebra of the 
$P$-independent sequence $\left(\log \rho^{(n)}, n \geqq 1\right)$. By the ordinary 0-1 law [5, p. 229] we conclude $\Theta$ is $P$-trivial. Exactly the same argument applies for $Q$.

J. Feldman's Theorem. Suppose $T$ is a set (the time domain), $\mathcal{R}$ is the set of real numbers, $\Omega=\mathcal{R}^{T}, \mathcal{F}=\mathfrak{B}^{T}$ (product Borel $\sigma$-algebra). Then

$$
(P, Q \text { Gaussian measures on } \mathcal{F}) \Rightarrow(P \perp Q \text { or } P \equiv Q) \text {. }
$$

Proof. Let $X_{t}(f)=f(t), t \in T$, be the coordinate random variables defined for $f \in R^{T}$. Suppose there is an $A \in \mathfrak{B}^{T}$ with $0=Q(A)<P(A)$. Then $A$ is measurable [6, Corollary, p. 81] with respect to the $\sigma$-subalgebra generated by countable subfamily $\left\{X_{t_{1}}, X_{t_{2}}, \cdots\right\}$ of $\left\{X_{t}, t \in T\right\}$. Let $\widetilde{F}_{\infty}=\sigma\left\{X_{t_{1}}, X_{t_{2}}, \cdots\right\}$.

Define for each $n, \mathscr{F}_{n}=\sigma\left\{X_{t_{1}}, \cdots, X_{t_{n}}\right\}$. For each $n$, the dichotomy $P\left|\mathscr{F}_{n} \equiv Q\right| \mathscr{F}_{n}$ or $P\left|\mathscr{F}_{n} \perp Q\right| \mathscr{F}_{n}$ is a property of finite dimensional Gaussian distributions, so mutual absolute continuity $P\left|\mathscr{F}_{n} \equiv Q\right| \mathscr{F}_{n}$ may as well be assumed. For every $s, t \in T$ let

$$
\begin{array}{ll}
m_{1}(t)=\int_{\Omega} X(t) d P, & K_{1}(s, t)=\int_{\Omega} X(x) X(t) d P-m_{1}(s) m_{1}(t), \\
m_{2}(t)=\int_{\Omega} X(t) d Q, & K_{2}(s, t)=\int_{\Omega} X(s) X(t) d Q-m_{2}(s) m_{2}(t) .
\end{array}
$$

For each $n<\infty$ we define certain vectors and matrices by restriction to $T_{n}=\left\{t_{1}, \cdots, t_{n}\right\}$, e.g. $X_{n}=\left(X\left(t_{1}\right), \cdots, X\left(t_{n}\right)\right), K_{1, n}=\left[K_{1}\left(t_{i}, t_{j}\right) ; i, j \leqq n\right]$, etc. Mutual absolute continuity $P\left|\mathscr{F}_{n} \equiv Q\right| \mathcal{F}_{n}$ ensures for each $n<\infty$ that the rows of $K_{1, n}$ span the same linear submanifold of $\mathcal{R}^{n}$ as do the rows of $K_{2, n}$. For each $n<\infty, \rho_{n}=\left(d Q \mid \mathscr{F}_{n}\right) /\left(d P \mid \mathscr{F}_{n}\right)$ takes explicit form

$$
\rho_{n}=\frac{\left|K_{1, \bar{n}}\right|^{1 / 2}}{\left|K_{2, \bar{n}}\right|^{1 / 2}} \frac{\exp \left[-\frac{1}{2}\left(X-m_{2}\right)_{\bar{n}} K_{2, \bar{n}}^{-1}\left(X-m_{2}\right)^{\frac{\mathrm{tr}}{n}}\right.}{\exp \left[-\frac{1}{2}\left(X-m_{1}\right)_{\bar{n}} K_{1, \bar{n}}^{-1}\left(X-m_{1}\right)_{\bar{n}}^{\mathrm{tr}}\right]}
$$

where $\bar{n}$ is the largest integer less than or equal to $n$ with $\left|K_{1, \bar{n}}\right|>0$. Rearrangement yields

$$
\begin{aligned}
\rho_{n}= & \frac{\left|K_{1, \bar{n}}\right|^{1 / 2}}{\left|K_{2, \bar{n}}\right|^{1 / 2}} \exp \left[-\frac{1}{2}\left(X-m_{2}\right)_{\bar{n}}\left(K_{2, \bar{n}}^{-1}-K_{1, \bar{n}}^{-1}\right)\left(X-m_{2}\right)_{\bar{n}}^{\operatorname{tr}}\right] \\
& \times \exp \left[\left(X-m_{1}\right)_{\bar{n}} K_{1, \bar{n}}^{-1}\left(m_{2}-m_{1}\right)_{\bar{n}}^{\mathrm{tr}}-\frac{1}{2}\left(m_{2}-m_{1}\right)_{\bar{n}} K_{1, \bar{n}}^{-1}\left(m_{2}-m_{1}\right)_{\bar{n}}^{\mathrm{tr}}\right] .
\end{aligned}
$$

In the reproducing kernel notation of [7],

$$
\begin{aligned}
\rho_{n}=\left|K_{2, \bar{n}}^{-1} K_{1, \bar{n}}\right|^{1 / 2} & \exp \frac{1}{2}\left(\left(X-m_{2}\right) \otimes\left(X-m_{2}\right), K_{2}-K_{1}\right)_{K_{1}, \bar{n} \otimes K_{2, \bar{n}}} \\
& \times \exp \left\{\left(X-m_{1}, m_{2}-m_{1}\right)_{K_{1, \bar{n}}}-\frac{1}{2}\left\|m_{2}-m_{1}\right\|_{K_{1}, \bar{n}}^{2}\right\} .
\end{aligned}
$$


If $n<\infty$, define $\mathcal{H}_{n}$ as the set of all real functions on $T$ which are finite real linear combinations of $\left\{K\left(\cdot, t_{1}\right), \cdots, K_{1}\left(\cdot, t_{n}\right)\right\}, \mathcal{M}_{\infty}=\bigcup_{n} \mathcal{M}_{n}$. Since $\mathcal{H}_{\infty}$ is dense in the reproducing kernel Hilbert space of the restriction $\left[K_{1}\left(t_{i}, t_{j}\right), i, j<\infty\right], P$-triviality of $\mathcal{G}$ can be established using the following 0-1 law for Gaussian processes:

Zero-one law. If $S$ is a set, $\left(\mathcal{R}^{S}, \mathcal{B}^{S}, P\right)$ a probability function space, $P$ a Gaussian measure, $A$ an event in the $P$-completion of $\mathcal{B}^{S}, \mathcal{D}$ a dense subset of the reproducing kernel Hilbert space of the covariance kernel (e.g. $\left.K_{1}\right)$ of $P$, then

$$
(e \in \mathfrak{D} \Rightarrow A=A+c) \Rightarrow(P(A)=0 \text { or } 1) .
$$

The proof will not be given here, but uses [3, Lemma 4] much as [3, Lemma 6]. This 0-1 law as applied to $\mathcal{G}$ asserts that $\mathcal{G}$ is $P$-trivial if invariant under the mapping $\left\{X\left(t_{j}\right) \rightarrow X\left(t_{j}\right)+e\left(t_{j}\right), j<\infty\right\}$ for every $e \in \mathcal{H}_{\infty}$. Such is indeed the case since if $e \in \mathcal{M}_{n}, k \geqq n$, simple calculation verifies that $\log \rho_{k+1}-\log \rho_{k}$ is unchanged when $X_{k+1}$ is replaced by $(X+e)_{k+1}$ and $X_{k}$ by $(X+e)_{k}$. Applying the Theorem, $P\left|\mathcal{F}_{\infty} \perp Q\right| \mathcal{F}_{\infty}$ or $P\left|\mathscr{F}_{\infty} \ll Q\right| \mathscr{F}_{\infty}$. Now $0=Q(A)<P(A)$ precludes $\ll$, hence $P \mid \mathscr{F}_{\infty} \perp$ $Q \mid \mathcal{F}_{\infty}$. Therefore (not $P \ll Q$ ) has been shown to imply $P \perp Q$. Interchanging the roles of $P, Q$ we obtain $P \equiv Q$ or $P \perp Q$.

An effective test for singularity was provided by Kakutani in the product measure case, and later completely generalized by Kraft [4] who proved that

$$
\left(\int_{\Omega} \rho_{n}^{1 / 2} d P \rightarrow 0\right) \Leftrightarrow(P \perp Q) .
$$

\section{REFERENCES}

1. J. Feldman, Equivalence and perpendicularity of Gaussian processes, Pacific J. Math. 8 (1958), 699-708. MR 21 \#1546.

2. S. Kakutani, On equivalence of infinite product measures, Ann. of Math. (2) 49 (1948), 214-224. MR 9, 340.

3. G. Kallianpur, Zero-one laws for Gaussian processes, Trans. Amer. Math. Soc. 149 (1970), 199-211.

4. Charles Kraft, Some conditions for consistency and uniform consistency of statistical procedures, Univ. Calif. Publ. Statist. 2 (1955), 125-141. MR 17, 505.

5. M. Loève, Probability theory, 3rd ed., Van Nostrand, Princeton, N.J., 1963. MR 34 \#3596.

6. J. Neveu, Bases mathématiques du calcul des probabilités, Masson, Paris, 1964; English transl., Holden-Day, San Francisco, Calif., 1965. MR 33 \#6659; \#6660.

7. E. Parzen, Probability density functionals and reproducing kernel Hilbert spaces, Proc. Sympos. Time Series Analysis (Brown University, 1962), Wiley, New York, 1963, pp. 155-169. MR 26 \#7119.

Department of Statistics and Probability, Michigan State University, East LANSING, MichigAN 48823 